\title{
Strong damping of phononic heat current by magnetic excitations in $\mathrm{SrCu}_{2}\left(\mathrm{BO}_{3}\right)_{2}$
}

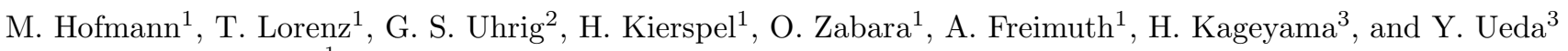 \\ ${ }^{1}$ II. Physikalisches Institut, Universität zu Köln, 50937 Köln, Germany \\ ${ }^{2}$ Institut für Theoretische Physik, Universität zu Köln, 50937 Köln, Germany \\ ${ }^{3}$ Institute for Solid State Physics, University of Tokyo, Kashiwanoha 5-1-5, Kashiwa, Chiba 27r-8581, Japan
}

(May 8, 2001)

\begin{abstract}
Measurements of the thermal conductivity as a function of temperature and magnetic field in the $2 \mathrm{D}$ dimer spin system $\mathrm{SrCu}_{2}\left(\mathrm{BO}_{3}\right)_{2}$ are presented. In zero magnetic field the thermal conductivity along and perpendicular to the magnetic planes shows a pronounced double-peak structure as a function of temperature. The low-temperature maximum is drastically suppressed with increasing magnetic field. Our quantitative analysis reveals that the heat current is due to phonons and that the double-peak structure arises from pronounced resonant scattering of phonons by magnetic excitations.
\end{abstract}

During the last few years the thermal conductivity $(\kappa)$ of low dimensional spin systems has attracted considerable interest [1] 6 . One reason is that in these materials a large magnetic contribution $\kappa^{m a g}$ to the heat current may be present as observed e.g. for the spin ladder material $\mathrm{Sr}_{14-x} \mathrm{Ca}_{x} \mathrm{Cu}_{24} \mathrm{O}_{41}$ [6]. Another reason is that the phononic heat current $\kappa^{p h}$ probes the spectrum of magnetic excitations as well as the spin-phonon coupling [3 5 . The latter is very important in some of the low dimensional spin systems, e.g. in the spin-Peierls compound $\mathrm{CuGeO}_{3}$ 迎. 阿. Both issues, the magnetic contribution to the heat current as well as the interaction of the phonons with magnetic excitations, are to a large extent unexplored and not well understood.

A material of particular interest in this context is $\mathrm{SrCu}_{2}\left(\mathrm{BO}_{3}\right)_{2}$ (SCBO) [7]. The $\mathrm{Cu}^{2+}$ ions form a quasi$2 \mathrm{D}$ spin system which is by virtue of the crystal geometry an experimental realization of the Shastry-Sutherland model [8]. In SCBO the intra-dimer and inter-dimer couplings are of magnitude $J_{1} \simeq 72 \mathrm{~K}$ and $J_{2} \simeq 43 \mathrm{~K}$, i.e. $J_{2} / J_{1} \simeq 0.6$ [9]. As expected for this ratio SCBO has a dimerized singlet ground-state separated from the excited triplet states by a finite gap $\Delta \simeq 35 \mathrm{~K}$ as seen in the magnetic susceptibility [10] or in inelastic neutron scattering [11]. From the latter it is also known that the triplet excitations are almost dispersionless, i.e the group velocity is very small, in agreement with theoretical calculations 12 15. Thus, a sizeable $\kappa^{m a g}$ is not expected for SCBO making this material a natural candidate to study the influence of magnetic excitations on $\kappa^{p h}$.

In this letter we present measurements of the thermal conductivity of SCBO along $\left(\kappa_{a}\right)$ and perpendicular $\left(\kappa_{c}\right)$ to the $2 \mathrm{D}$ spin planes in a large temperature $(2.5-275 \mathrm{~K})$ and magnetic field range $(0-17 \mathrm{~T})$. In zero field, both $\kappa_{a}$ and $\kappa_{c}$ show pronounced double-peak structures as a function of $T$. For both directions the low- $T$ maximum is drastically suppressed by a magnetic field. We present a model based on a purely phononic thermal conductivity and explain the double-peak structure and its field dependence by strong damping of the phononic heat current due to resonant scattering of phonons by magnetic excitations. With the same parameters our model considerably improves the description of the magnetic field dependence of the specific heat of SCBO [16].

For our study two samples of rectangular-bar-shaped form of $\sim 0.6 \times 1.9 \times 3 \mathrm{~mm}^{3}$ with the long direction along the $a$ and $c$ axis, respectively, were cut from larger single crystals of SCBO grown by the traveling solvent floating zone method [17]. The thermal conductivity was measured with a conventional steady state method using differential Chromel-Au $+0.07 \%$ Fe thermocouples calibrated in magnetic fields. Typical temperature gradients were of the order of $0.2 \mathrm{~K}$. The absolute accuracy of our measurements is of order $\pm 10 \%$ because of uncertainties in the sample geometry whereas the relative accuracy is about one order of magnitude better [19].

We show in Fig. 1 the thermal conductivity $\kappa_{a}(T)$ $\left(=\kappa_{b}\right.$ in tetragonal SCBO $)$ and $\kappa_{c}(T)$ in zero magnetic field. For both directions pronounced double-peak structures are observed with the low $T$ maxima occurring at $\approx 4.5 \mathrm{~K}$. For $\kappa_{a}$ the high $T$ maximum lies at $\approx 60 \mathrm{~K}$ and for $\kappa_{c}$ at $\approx 30 \mathrm{~K}$. Above $100 \mathrm{~K}$ we find $\kappa_{c} \propto T^{-1}$ as expected for phonon heat transport [20] whereas $\kappa_{a}$ follows a $T^{-0.6}$ dependence. The magnetic field dependence of the thermal conductivity is shown in Fig. 2. The low $T$ maximum is suppressed strongly by a magnetic field for both $\kappa_{a}$ and $\kappa_{c}$. The magnetic field dependence at higher temperatures is only weak $(\leq 2 \%)$ and close to the relative measurement accuracy. The minimum of the double-peak structure and the low $T$ maximum systematically shift to lower temperatures with increasing magnetic field.

The behavior of $\kappa$ observed here is reminiscent of that found in other low dimensional spin systems [1, 4, 6 . In particular, in $\mathrm{CuGeO}_{3} \kappa$ also shows an almost fieldindependent maximum at $\sim 20 \mathrm{~K}$ and a second one around $5 \mathrm{~K}$, which is strongly suppressed by a magnetic field. This has been interpreted in terms of a magnetic 


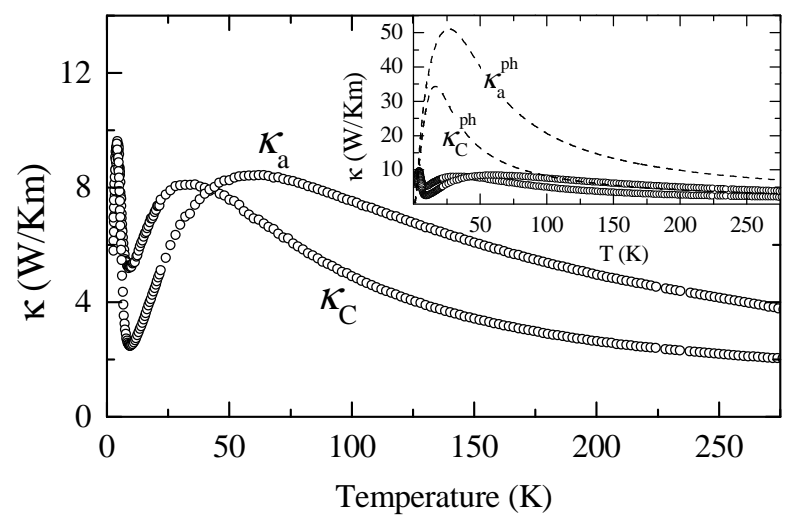

FIG. 1. Thermal conductivity of $\mathrm{SrCu}_{2}\left(\mathrm{BO}_{3}\right)_{2}$ along $\left(\kappa_{a}\right)$ and perpendicular $\left(\kappa_{c}\right)$ to the magnetic planes. The inset shows the experimental data (o) together with "conventional" phononic contributions $\kappa_{a}^{p h}$ and $\kappa_{c}^{p h}(--)$ obtained by switching off the scattering of phonons by magnetic excitations (see text).

and a phononic contribution to the heat current with maxima at different temperatures [4].

For several reasons, a similar interpretation is not possible in SCBO. Firstly, due to their flat dispersion the single triplet excitations are not expected to contribute significantly to the heat current. Secondly, $\kappa^{m a g}$ in a 2D magnetic system should be strongly anisotropic, as is observed e.g. in $\mathrm{Sr}_{14-x} \mathrm{Ca}_{x} \mathrm{Cu}_{24} \mathrm{O}_{41}$ [6], but not in our measurements. The lack of a strong anisotropy excludes also an explanation in terms of multi-triplet excitations observed in SCBO above $\sim 5 \mathrm{meV}$ 11, 16, 21, 22]. These excitations move much more easily than the single triplet excitations [9], but again only within the magnetic planes. Finally, the relation $\kappa \propto c v \ell$ ( $c$ is the specific heat, $v$ the group velocity and $\ell$ the mean free path) predicts similar $T$ dependencies of $\kappa^{m a g}$ and the magnetic specific heat $c^{m a g}$. But measurements of $c$ in fields up to $12 \mathrm{~T}$ [16] show that $c^{\text {mag }}$ gives rise to a maximum of $c / T$ at temperatures close to the minima of $\kappa_{a}$ and $\kappa_{c}$ for the respective magnetic fields (see Fig. 2).

From these arguments we exclude a sizable magnetic contribution to the heat current [18], so that $\kappa \approx \kappa^{p h}$. As the cause of the double peak structure and its magnetic field dependence we propose scattering of phonons by magnetic excitations. The absence of a strong anisotropy of $\kappa_{a}$ and $\kappa_{c}$ is then easily understood, since both, the anisotropy of the spin-phonon coupling and/or of the phonon system itself are expected to be much weaker than that of the 2D magnetic system. In addition, this scenario yields a straightforward explanation why the minima of $\kappa_{a}$ and $\kappa_{c}$ occur at the maxima of $c / T$. The latter is the temperature derivative of the magnetic entropy and thus directly related to the number of magnetic excitations which serve as scatterers for the phonons.

For a quantitative description we fit our data by a Debye model for the phononic thermal conductivity [23]
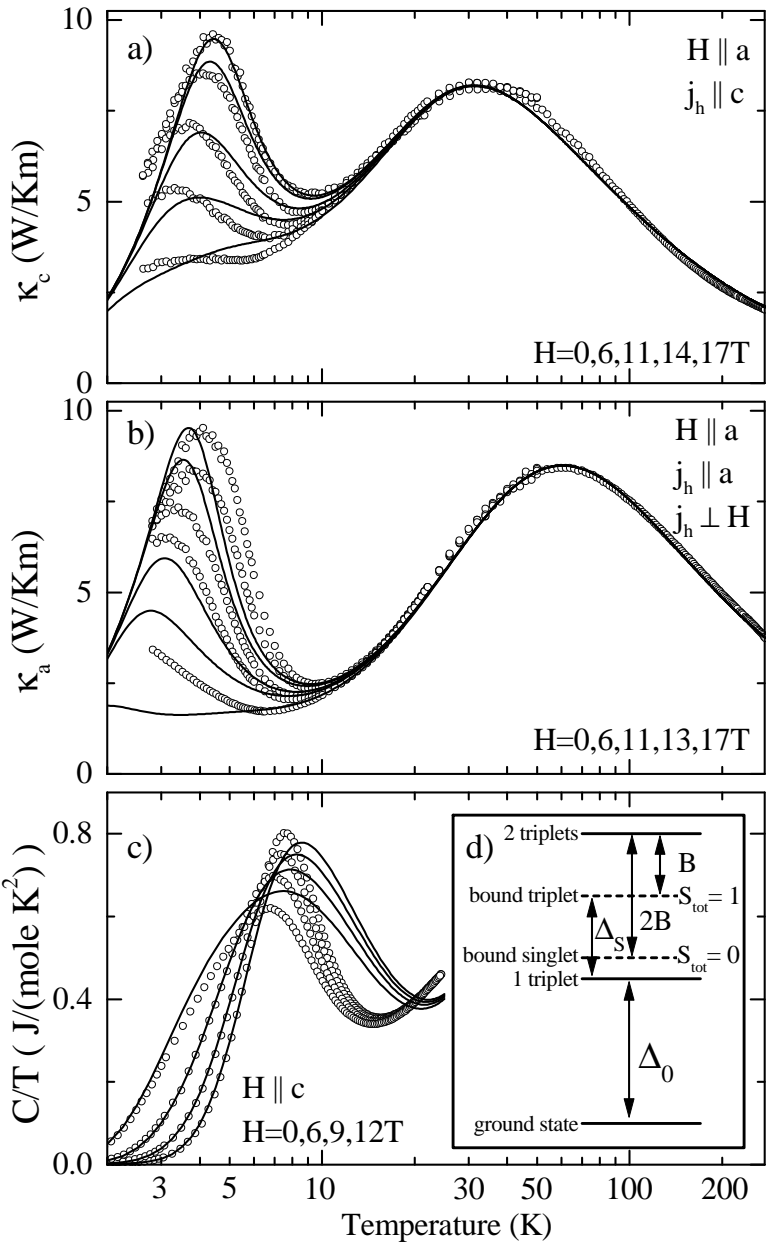

FIG. 2. Thermal conductivity $\kappa_{c}$ (a) and $\kappa_{a}$ (b) on a logarithmic temperature scale for various magnetic fields (see figure). On increasing field the low- $T$ maximum is suppressed. The lines are theoretical curves calculated for the same magnetic fields via Eq. (11). (c) Specific heat at various fields $H \| c$ (the peak height decreases with increasing field). Data points are from Ref. [16]; the lines result from our theory. (d) Level scheme used in our model: The solid lines denote the energies of the ground state, 1 triplet and 2 elementary triplets, respectively. The dashed lines indicate bound states with $S_{t o t}$ $=0$ or 1 , which are built from two elementary triplets. $B$ is the binding energy and $\Delta_{0}$ is the elementary gap. $\Delta_{s}$ is the energy difference relevant for the resonance scattering according to Eq. (3).

$$
\kappa^{p h}=\frac{k_{B}}{2 \pi^{2} v}\left(\frac{k_{B}}{\hbar}\right)^{3} T^{3} \int_{0}^{\Theta_{D} / T} \frac{x^{4} e^{x} \tau(\omega, T)}{\left(e^{x}-1\right)^{2}} d x,
$$

where $v$ is the sound velocity, $\Theta_{D}$ the Debye temperature, $\omega$ the phonon frequency, $x=\hbar \omega / k_{B} T$ and $\tau(\omega, T)$ the mean lifetime of a phonon. The total scattering rate $\tau^{-1}$ is given by

$$
\tau^{-1}=\tau_{b d}^{-1}+\tau_{p t}^{-1}+\tau_{u m}^{-1}+\tau_{\text {res }}^{-1} .
$$

Here $\tau_{b d}^{-1}, \tau_{p t}^{-1}$, and $\tau_{u m}^{-1}$ refer to the standard relaxation processes for conventional phonon heat transport, i.e. 
to boundary scattering, scattering by point defects, and umklapp scattering, respectively [24. $\tau_{\text {res }}^{-1}$ describes the scattering of phonons on magnetic excitations, which will be considered in the following.

For the double-peak structure of the phononic thermal conductivity we need a scattering mechanism that is most active in a certain temperature range. We therefore consider resonant scattering [23,25 27]. The physical picture is the following: A phonon of energy $\omega$ is absorbed, thereby exciting the magnetic system (under the constraint of spin conservation). Then another phonon of the same energy is emitted and the magnetic system is deexcited. If excitation (or deexcitation) starts from a localized state like the dispersionless triplet excitations of SCBO there is no directional correlation between the absorbed and the emitted phonon. The net effect is therefore the same as that of point-like defects except for the frequency dependence of the matrix element $M_{k^{\prime}, k}(\omega)$, and the scattering rate $\tau_{\text {res }}^{-1}$ is of the form

$$
\tau_{\text {res }}^{-1}(\omega)=C \omega^{2} M_{k^{\prime}, k}^{2}(\omega) F(T)=C \frac{\omega^{4} \Delta_{s}^{2}}{\left(\omega^{2}-\Delta_{s}^{2}\right)^{2}} F(T) .
$$

Here, $\Delta_{s}$ is the energy splitting of the two levels involved, $C$ is the overall coupling strength between phonons and the two-level system and $F(T)$ contains information on the state of the two-level system 23,25,26. Notably, resonant scattering starting from the magnetic ground state is not effective! The reason is that the ground state is a coherent, translational invariant state with zero momentum so that the emitted and absorbed phonons must have the same momentum and energy.

With this scenario in mind we consider resonant scattering within the magnetic excitation spectrum of SCBO (Fig. 2d). The lowest excited state is a single triplet with excitation energy $\Delta_{0}$. It is thermally excited with probability $c_{1}=1-\left[1+m_{1} \exp \left(-\beta \Delta_{0}\right)\right]^{-1}$. Here $\beta=\left(k_{B} T\right)^{-1}$ and $m_{1}=1+2 \cosh (\beta h)$ contains the magnetic field dependence through $h=g \mu_{B} H$. In the resonance process a second triplet is excited on one of the two adjacent dimers, and it combines with the first, thermally excited triplet to a bound triplet state so that the total spin is conserved. The energy of the bound triplet is given by $2 \Delta_{0}-B$, where $B$ is the binding energy 28 . The resonance energy to be used in Eq. (3) is therefore $\Delta_{s}=\left(2 \Delta_{0}-B\right)-\Delta_{0}=\Delta_{0}-B$. A complete description of the thermal factors of the resonance process takes account of (i) the fact that a thermally excited triplet has to be present and (ii) of all resonance processes involving the 2 adjacent dimers. This yields 19

$$
F(T)=2 c_{1}\left(N_{0}+N_{1}+\left(N_{0}-N_{1}\right)^{2}\right)
$$

with the probabilities $N_{0}=1-c_{1}$ of having a dimer in its singlet state and $N_{1}=c_{1} / 3$ of having a dimer in the triplet state with a particular $S^{z}$ component. Note that in the resonance process the total $S^{z}$ component is conserved due to spin conservation. Therefore $\Delta_{s}$ does not depend on the magnetic field. It is only $c_{1}$ that is field dependent.

In zero field Eqs. (3, 4) yield an excellent fit (not shown) for $\Delta_{0} \approx 35 \mathrm{~K}$ and $\Delta_{s} \approx 20 \mathrm{~K}$ indicating strong binding effects $(B \approx 15 \mathrm{~K})$. This is in good agreement with inelastic neutron scattering and ESR experiments showing the lowest bound triplets at $\approx 55 \mathrm{~K}$ and an elementary gap of $\Delta_{0} \approx 35 \mathrm{~K}$ (see Fig. 2d d) [11,21]. However, the decrease of the thermal conductivity in magnetic fields computed by Eqs. (3, 4) is much stronger than that found in our experiments.

We can account for this discrepancy by considering a second, magnetic field independent contribution to the resonance scattering. It arises from the presence of bound singlet excitations with energy $2 \Delta_{0}-2 B$ [9,28] (see Fig. $2 \mathrm{~d}$ ). Since $2 B \approx \Delta_{0}$ in SCBO, the energy of these bound singlets is close to that of single triplet excitations yielding comparable thermal populations of these states. Therefore bound singlets have to be treated as scattering centers for resonance scattering too. In this case a dimer adjacent to a bound singlet is excited and the corresponding 3 triplets combine to $S_{t o t}=0$. Since $S_{t o t}=0$, this contribution to the resonance scattering does not depend on a magnetic field and lowers the sensitivity of the total resonance scattering rate to a magnetic field. The contribution of the bound singlets to the scattering rate is treated in analogy to that of the single triplets. The probability that a pair of adjacent dimers is in a state made of two triplets is given by $c_{2}=1-\left(1+2 m_{1} \exp \left(-\beta \Delta_{0}\right)\right) / Z$, where $Z$ is the complete partition sum of the system of two triplets. In this way we achieve a theoretical description comprising single triplets with density per dimer $n_{1}$ and 2-triplet states with density per dimer $n_{2}$. Exclusion effects - a dimer cannot be in a single triplet state and involved in one of the bound states simultaneously - are accounted for by $n_{1}=c_{1}\left(1-4 n_{1}-2 n_{2}\right)$ and $n_{2}=2 c_{2}\left(1-2 n_{1}-3 n_{2}\right)$.

A complication arises from a repulsive interaction $v$ between neighboring triplets. The energy $\Delta_{0}$ of a single triplet is smaller than $J_{1}$ due to virtual processes between neighboring dimers resulting from the dimerdimer coupling. If a triplet is excited on a neighboring dimer these processes are (in part) blocked and $\Delta_{0}$ increases. Accounting for this on a mean-field level yields $v=R\left(n_{1}+3 n_{2} / 2\right)$ with $R=\left(J_{1}-\Delta_{0}\right) / 2$ [19]. In the calculation of $\kappa$ one has to replace $\Delta_{0} \rightarrow \Delta_{0}+p v$ in all equations (i.e. in $c_{1}, c_{2}$ and the energies given in [28]), where $p=2$ refers to single triplets and $p=3 / 2$ to bound states from two triplets.

To illustrate the validity of our model we compute the specific heat for various magnetic fields. For the realistic parameters $\Delta_{0}=36 \mathrm{~K}, R=18 \mathrm{~K}$ (from $J_{1}=72 \mathrm{~K}$ [9]) and $B=17 \mathrm{~K}$ we obtain the results depicted in Fig. 2cc. At low $T$ the agreement is very good; position and height 
of the peak are considerably improved relative to the isolated dimer model [16]. At higher $T$ we presume that the local model developed above is too simplistic since the dispersion [9,11] of the bound states is neglected.

The resonant scattering, decisive for the thermal conductivity, is calculated in the same model with the same parameters. We obtain $\tau_{r e s}^{-1}=C \omega^{4}\left(A_{1}+A_{2}\right)$ with

$$
\begin{aligned}
& A_{1}=\frac{2 n_{1}\left(N_{0}+N_{1}+\left(N_{0}-N_{1}\right)^{2}\right)\left(\Delta_{0}-B\right)^{2}}{\left(\left(\Delta_{0}-B\right)^{2}-\omega^{2}\right)^{2}} \\
& A_{2}=\frac{3 n_{2}\left(N_{0}+N_{1}+2\left(N_{0}-N_{1}\right)^{2}\right) \Delta_{0}^{2}}{\left(\Delta_{0}^{2}-\omega^{2}\right)^{2}}
\end{aligned}
$$

where $N_{1}=\left(n_{1}+3 n_{2} / 2\right) / 3$ and $N_{0}=1-3 N_{1}$. Using this $\tau_{\text {res }}^{-1}$ together with the usual scattering rates (Eq. (2)) we fit the experimental $\kappa_{a}$ and $\kappa_{c}$ by Eq. (11). The sound velocity $(v=7600 \mathrm{~m} / \mathrm{s})$ along the a-direction and $\Theta_{D}=453 \mathrm{~K}$ are calculated from the measured elastic constant $c_{11}$ 29]. Due to the lack of experimental data for $c_{33}$ we take the same velocity for the fit of $\kappa_{c}$. The relaxation rate $\tau_{b d}^{-1}$ is then obtained by $\tau_{b d}^{-1}=v / L$ with the characteristic sample lengths $L=0.75 \mathrm{~mm}\left(\kappa_{a}\right)$ and $0.5 \mathrm{~mm}\left(\kappa_{c}\right)$ of the two samples. The point defect scattering rate is given by $\tau_{p t}^{-1}=P \omega^{4}$ and the phonon umklapp scattering rate is approximated by $\tau_{u m}^{-1}=U T \omega^{3} \exp \left(\Theta_{D} / u T\right)$ 24. Since the gap $\Delta_{0}$, the binding energy $B$, and the interaction $R$ are determined from the specific heat there remain only four adjustable parameters $(P, U, u$, and $C)$.

As shown in Fig. 2 our model yields an almost perfect fit of the measured $\kappa_{c}$ (for $H=0$ ) over the entire $T$ range. Above $10 \mathrm{~K}$ the fit of $\kappa_{a}$ is also almost perfect, whereas below $10 \mathrm{~K}$ a slight $T$ shift $(\leq 1 \mathrm{~K})$ between fit and experimental data occurs. We use $P=7.7 \cdot 10^{-43} \mathrm{~s}^{3}$ $\left(7.9 \cdot 10^{-43} \mathrm{~s}^{3}\right), U=2.6 \cdot 10^{-31} \mathrm{~s}^{2} / \mathrm{K}\left(5.7 \cdot 10^{-31} \mathrm{~s}^{2} / \mathrm{K}\right)$, $u=5(10)$, and $C=3.35 \cdot 10^{6} \mathrm{~s}^{3}\left(1.15 \cdot 10^{6} \mathrm{~s}^{3}\right)$ for the calculation of $\kappa_{a}\left(\kappa_{c}\right)$. These values are reasonable for an insulator and are comparable in magnitude to those found e.g. in the spin ladders $[6]$. Different values for $\kappa_{a}$ and $\kappa_{c}$ are expected in an anisotropic crystal.

Accounting for the Zeeman splitting in $c_{1}$ and $c_{2}$, we calculate $\kappa_{a}$ and $\kappa_{c}$ in magnetic fields without further parameter adjustment. Using $g \approx 2.07$ [10], our model reproduces the overall influence of the magnetic field very well. The high $T$ maxima remain unchanged whereas the low $T$ maxima are continuously suppressed. Again the agreement between calculated and experimental values is better for $\kappa_{c}$ than for $\kappa_{a}$. Whereas the field influence on $\kappa_{a}$ is slightly overestimated by our model the experimental values of $\kappa_{c}$ are even quantitatively reproduced up to the highest field.

In the calculations, one can switch off the resonant scattering on magnetic excitations by setting $C=0$. $\kappa^{p h}(C=0)$ obtained in this way is much larger than the measured thermal conductivity, as shown in the inset of Fig. 1. As expected for resonant scattering the damp- ing of the phonon heat transport is most pronounced for $T \approx \Delta_{s}$. Note, however, that even at room temperature the suppression is sizeable. This strong damping gives further evidence for a large spin phonon coupling in SCBO as has been inferred before from sound wave anomalies [29].

In summary, the thermal conductivity of the low dimensional quantum spin system $\mathrm{SrCu}_{2}\left(\mathrm{BO}_{3}\right)_{2}$ has a characteristic double-peak structure with two maxima at low temperatures and a pronounced magnetic field dependence. A significant magnetic contribution to the heat current can be excluded, since the double peak structure is not anisotropic and because the magnetic excitations are (almost) localized. Our quantitative analysis in terms of resonant scattering of phonons on magnetic excitations explains the double peak structures and their magnetic field dependence very well and gives evidence for strong spin-phonon coupling. Therefore, we call for further investigations whether the same mechanism is at work in other low-dimensional spin systems.

We acknowledge useful discussions with E. MüllerHartmann, M. Grüninger and A. P. Kampf. This work was supported by the Deutsche Forschungsgemeinschaft in SFB 341 and SP 1073.

[1] Y. Nakamura et al., Physica 185-189, 1409 (1991).

[2] J. L. Cohn et al., Phys. Rev. B 52, R13134 (1995).

[3] O. Baberski et al., Europhys. Lett. 44, 335 (1998).

[4] Y. Ando et al., Phys. Rev. B 58, R2913 (1998).

[5] A. N. Vasil'ev et al., Phys. Rev. Lett. 81, 1949 (1998).

[6] A. V. Sologubenko et al., Phys. Rev. Lett. 84, 2714 (2000).

[7] H. Kageyama et al., Phys. Rev. Lett. 82, 3168 (1999).

[8] B. S. Shastry and B. Sutherland, Physica 108B, 1069 (1981).

[9] C. Knetter et al., Phys. Rev. Lett. 85, 3958 (2000).

[10] H. Kageyama et al., J. Phys. Soc. Jpn. 68, 1821 (1999).

[11] H. Kageyama et al., Phys. Rev. Lett. 84, 5876 (2000).

[12] S. Miyahara and K. Ueda, Phys. Rev. Lett. 82, 3701 (1999).

[13] Z. Weihong et al., Phys. Rev. B 60, 6608 (1999).

[14] E. Müller-Hartmann et al., Phys. Rev. Lett. 84, 1808 (2000).

[15] C. Knetter et al., J. Phys. Cond. Mat. 12, 9069 (2000).

[16] H. Kageyama et al., Sov. Phys. JETP 90, 129 (2000).

[17] H. Kageyama et al., J. Crystal Growth 206, 65 (1999).

[18] A small magnetic contribution to $\kappa_{a}$ can of course not be excluded. Such a contribution might explain in part the different temperature dependences of $\kappa_{a}$ and $\kappa_{c}$, but it is unimportant in the context of the double peak structure.

[19] Details will be presented in a forthcoming publication.

[20] R. Bermann, Thermal Conduction in Solids (Clarendon Press, Oxford, 1976). 
[21] H. Nojiri et al., J. Phys. Soc. Jpn. 68, 2907 (1999).

[22] P. Lemmens et al., Phys. Rev. Lett. 85, 2605 (2000).

[23] Neelmani and G. S. Verma, Phys. Rev. B 6, 3509 (1972).

[24] P. G. Klemmens, in Solid State Physics, edited by H. Ehrenreich, F. Seitz, and D. Turnbull (Academic, New York, 1958), Vol. 7.

[25] V. Roundy and D. L. Mills, Phys. Rev. B 1, 3703 (1970).

[26] G. A. Toombs and F. W. Sheard, J. Phys. Cond. Mat. 6, 1467 (1973).
[27] The more conventional scattering mechanisms where e.g. a phonon decays into two magnetic excitations are far less effective, basically because of the discreteness of the magnetic excitation spectrum.

[28] The coupling $B \mathbf{S}_{\mathrm{a}} \mathbf{S}_{\mathrm{b}}$ between two triplets on adjacent dimers leads to the total energies $2 \Delta_{0}-2 B, 2 \Delta_{0}-B$ and $2 \Delta_{0}+B$ for $S_{t o t}=0,1$, and 2 , respectively.

[29] S. Zherlitsyn et al., Phys. Rev. B 62, 6097 (2001). 